\title{
Topological Charge Correlators, Spectral Bounds, and Contact Terms *
}

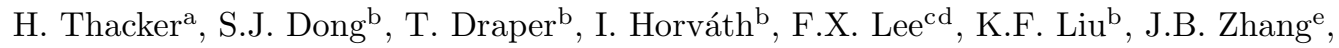 \\ ${ }^{a}$ Dept. of Physics, University of Virginia, Charlottesville, VA 22904 \\ ${ }^{\text {b} D e p a r t m e n t ~ o f ~ P h y s i c s ~ \& ~ A s t r o n o m y, ~ U n i v e r s i t y ~ o f ~ K e n t u c k y, ~ L e x i n g t o n, ~ K Y ~ 40506, ~ U S A ~}$ \\ ${ }^{\mathrm{c}}$ Center for Nuclear Studies, George Washington University, Washington, DC 20052, USA \\ d Jefferson Lab, 12000 Jefferson Avenue, Newport News, VA 23606, USA \\ ${ }^{\mathrm{e}} \mathrm{CSSM}$ and Dept. of Physics and Math. Physics, University of Adelaide, Adelaide, SA 5005, Australia
}

The structure of topological charge fluctuations in the QCD vacuum is strongly restricted by the spectral negativity of the Euclidean correlator for $x \neq 0$ and the presence of a positive contact term. Some examples are considered which illustrate the physical origin of these properties.

\section{Introduction}

Although topological charge plays a fundamental role in our understanding of low-energy hadron physics, the detailed structure of topological charge fluctuations in the QCD vacuum is not well understood. The construction of a local topological charge density operator for QCD [1] in terms of a Dirac operator with GW symmetry [2] has made it possible not only to study local $q(x)$ distributions in Monte Carlo generated gauge fields, but to analyze these distributions in terms of an eigenmode expansion for the corresponding Dirac operator. As discussed in [3], the resulting "eigenmode filtered" densities provide a physically meaningful way of removing shortwavelength background fluctuations and focusing on whatever longer range structures might appear. The necessity for some such filtering procedure is made clear by a fundamental property of the two-point correlator in Euclidean 4-space $\llbracket$, namely, that it must be negative at any nonzero separation,

$G(x) \equiv\langle q(x) q(0)\rangle \leq 0$ for $|x| \neq 0$

This follows from reflection positivity (because $q(x)$ is reflection odd), or equivalently, from spec-

*Talk presented by H. Thacker tral positivity in Minkowski space. In the latter derivation, the negative sign of the Euclidean correlator arises from the fact that $\mathbf{B}$ fields remain real under Euclidean rotation but $\mathbf{E}$ fields acquire a factor of $i$. The bound (1) places important restrictions on any realistic picture of topological charge in the QCD vacuum. For any nonzero separation, the positive contributions to the correlator from coherent, finite-size fluctuations of topological charge (e.g. instantons) must necessarily be overwhelmed by anti-correlated background fluctuations. Moreover, the requirement that the topological susceptibility $\chi_{t}=\int G(x) d^{4} x$ be positive implies that $\mathrm{G}(\mathrm{x})$ must include a positive contact term $\propto \delta^{4}(x)$ which makes the largest contribution to the $\chi_{t}$ integral.

The results of a numerical investigation of the topological charge correlator in QCD [3] indicate that it is very short range and consistent with being dominated, in the continuum limit, by an effective delta-function contact term. The fact that the topological charge correlator in QCD is approximately a delta-function can also be inferred from numerical studies of the quenched pseudoscalar hairpin correlator (i.e. the $\eta^{\prime}$ mass insertion diagram) [5]. The measured correlator fits extremely well at all time separations to the dipole form $\propto\left(1+m_{\pi} \tau\right) \exp \left(-m_{\pi} \tau\right)+(\tau \rightarrow T-\tau)$. 
This implies that the amputated diagram (which, in quenched QCD, is proportional to the topological charge correlator) has very little $q^{2}$ dependence and is approximately a delta-function in space-time.

In this talk, we will discuss some examples which illustrate how the negativity property (11) is satisfied in practice, and also consider the physical origin of the contact term. As a first example, consider the thermodynamics of a nonrelativistic free particle moving in one compact spatial dimension. [6] Denoting the spatial coordinate by $\phi$, the action is $S=\frac{1}{2} \dot{\phi}^{2}$. The partition function at inverse temperature $\beta$ is given by the Euclidean path integral over all paths satisfying

$\phi(\beta)=\phi(0)+C \nu$

where $C$ is the circumference of the compact dimension and $\nu$ is the winding number of the path. The winding number is the integral of a local topological charge density, $\nu=\int_{0}^{\beta} q(\tau) d \tau$ where

$q(\tau)=C^{-1} \dot{\phi}(\tau)$

There are classical n-instanton solutions which satisfy the Euclidean equation of motion,

$\phi_{n}(\tau)=\frac{C n}{\beta} \tau$

with action

$S_{n}=\frac{C^{2} n^{2}}{2 \beta^{2}}$

We decompose any path into the sum of an n-instanton solution and periodic fluctuations around it,

$\phi(\tau)=\phi_{n}(\tau)+\delta \phi(\tau)$

where $\delta \phi(\beta)=\delta \phi(0)$. Then it is easy to show that the topological charge correlator separates into a sum over instantons + oscillators,

$$
\begin{gathered}
G(\tau) \equiv\langle\dot{\phi}(\tau) \dot{\phi}(0)\rangle= \\
\frac{C^{2}}{\beta^{2}} \sum_{n} n^{2} e^{-\beta S_{n}} / \sum_{n} e^{-\beta S_{n}}+\langle\delta \dot{\phi}(\tau) \delta \dot{\phi}(0)\rangle
\end{gathered}
$$

The second term, coming from quantum fluctuations around the classical n-instanton solutions, is obtained by differentiating the free propagator

$\langle\delta \dot{\phi}(\tau) \delta \dot{\phi}(0)\rangle=-\frac{1}{\beta} \frac{\partial^{2}}{\partial \tau^{2}} \sum_{q_{j}} \frac{e^{-i q_{j} \tau}}{q_{j}^{2}+\lambda^{2}}$ where $q_{j}=\frac{2 \pi j}{\beta}$. Here $\lambda \rightarrow 0$ is a small infrared cutoff parameter. Thus, the oscillator contribution is

$\langle\delta \dot{\phi}(\tau) \delta \dot{\phi}(0)\rangle=\frac{1}{\beta} \sum_{j \neq 0} e^{-i q_{j} \tau}=\delta(\tau)-\frac{1}{\beta}$

Now let's consider how the correlator (7) satisfies the bound (11). There are two limiting cases of interest:

(I) Semiclassical or high temperature limit $(\beta \rightarrow$ 0 or $C \rightarrow \infty$ ). In this limit, the instanton expansion converges, but the terms are exponentially suppressed. The bound (1) is satisfied because the negative term $-1 / \beta$ from the quantum fluctuations (9) is always larger than the positive instanton contribution. In this case, if we introduce a $\theta$ term, the instanton expansion gives a good description of $\theta$ dependence (e.g. topological susceptibility).

(II) Ultra-quantum mechanical or low temperature limit $(\beta \rightarrow \infty$ or $C \rightarrow 0)$. In this case, the instanton sum diverges. Instead of expanding in winding number, the instanton series may be resummed by a Poisson transformation

$$
\sum_{n} e^{-n^{2} / \alpha}=\sqrt{\pi \alpha} \sum_{m} e^{-\alpha \pi^{2} m^{2}}
$$

Using this formula, we find, in the large $\beta$ limit, the resummed instanton expansion $\rightarrow+1 / \beta$ Thus, in this limit, the $-1 / \beta$ from quantum fluctuations exactly cancels the instanton contribution, leaving only the contact term,

$G(\tau) \rightarrow \delta(\tau)$

Note that, in case II, the expansion of the Poisson-resummed instanton series is in no sense an expansion in number of instantons, but is in fact dual to it. It's convergence corresponds to a breakdown of the usual instanton expansion. In some respects, this case may be viewed as a greatly oversimplified analog of Witten's picture of the QCD vacuum, in which topological susceptibility is finite, but is not properly described in terms of an instanton expansion.

Note the origin of the contact term in this $0+1$ dimensional example. In momentum space, the two factors of $q$ coming from the derivatives in 
the definition of the topological charge operators exactly cancel the $1 / q^{2}$ pole of the propagator. This is a manifestation of "vacuum seizing" [7], originally discussed in the Schwinger model as a possible mechanism for resolving the $\mathrm{U}(1)$ problem in QCD.

As a second example of a Euclidean topological charge correlator, we consider the $\mathrm{CP}(\mathrm{N}-1)$ sigma model in two space-time dimensions. We have studied the topological charge correlator via (1) the large $\mathrm{N}$ expansion, (2) a lattice strong coupling expansion, and (3) numerical Monte Carlo calculations. A complete discussion of this study will be presented elsewhere [8]. The results all indicate the dominance of the contact term in the TC correlator. First consider the large N expansion. It is well-known that, to leading order in large $\mathrm{N}$, the auxiliary $\mathrm{U}(1)$ gauge field develops a kinetic term and becomes dynamical due to scalar loop effects, thereby generating a long range (confining) Coulomb potential. Thus the gauge field correlator behaves like

$$
\int d^{2} x e^{i q \cdot x}\left\langle A_{\mu}(x) A_{\nu}(0)\right\rangle \approx \frac{1}{q^{2}}\left(-g_{\mu \nu}+\frac{q_{\mu} q_{\nu}}{q^{2}}\right)
$$

The corresponding Euclidean correlator for the topological charge operator $q(x)=\epsilon^{\mu \nu} \partial_{\mu} A_{\nu}$ thus produces a contact term,

$\int d^{2} x e^{i q \cdot x}\langle q(x) q(0)\rangle \approx$ const.

We have also studied the TC correlator for $\mathrm{CP}(\mathrm{N}-1)$ in a lattice strong-coupling expansion and by Monte Carlo simulation. For the timedependent correlator

$G\left(x_{0}\right)=\left.\int d x_{1}\langle q(x) q(0)\rangle\right|_{x_{0}=\tau}$,

we find that, for $\mathrm{CP}(1)$ in the region $0 \leq \beta \leq$ 2.0 (correlation length $\leq 35), G(\tau)$ is completely dominated by a contact term of the form $G(\tau)=$ $C_{0} \delta(\tau)+C_{2} \delta^{\prime \prime}(\tau)$, with $C_{0} \rightarrow \approx 0$ in the weak coupling region. Calculations for larger $\mathrm{N}$ models are in progress.

Based on these examples, one might suspect that a short range topological charge correlator dominated by a positive contact term is associated with a strong-coupling vacuum structure for which a description based on classical instanton solutions is inappropriate. In view of the recent QCD results [3] it is interesting to ask whether a theoretical mechanism exists for generating such a contact term in QCD. Using an operator product expansion, it is found that not only does the OPE predict the existence and approximate magnitude of the contact term [9], but the calculation itself closely resembles the vacuum seizing mechanism encountered in the simpler examples. The key point is that, because $\left\langle F^{2}\right\rangle \neq 0$ in the QCD vacuum, there is a term in the OPE for the $F \tilde{F}$ correlator where one gluon carries the large momentum $q$, while a pair of soft gluons (one from each source) disappears into the vacuum. Just as in the simpler examples, the $1 / q^{2}$ pole of this gluon propagator is cancelled by the momentum factors which arise from derivatives in the definition of the topological charge operator. A straightforward calculation shows that this effective one-gluon exchange graph gives a contact term $\propto \delta^{4}(x)$, and also $\propto\left\langle F^{2}\right\rangle$. Using the QCD sum rule estimate of $\left\langle F^{2}\right\rangle$, one finds [9] that this contact term makes a contribution to the $\eta^{\prime}$ mass of $m_{\eta^{\prime}} \approx \sqrt{\alpha_{S}} \times 400 \mathrm{MeV}$.

\section{REFERENCES}

1. P. Hasenfratz, V. Laliena, F. Niedermayer, Phys. Lett. B427, 125 (1998).

2. H. Neuberger, Phys. Lett. B417, 141 (1998).

3. I. Horváth et al, hep-lat/0203027, and I. Horvath, et al, these proceedings.

4. E. Seiler, I.O. Stamatescu, MPI-PAE/Pth $10 / 87$.

5. W. Bardeen, A. Duncan, E. Eichten, and H. Thacker, Phys. Rev. D62:114505 (2000), and E. Eichten, et al, these proceedings.

6. P. Arnold and L. McLerran, Phys. Rev. D37, 1020 (1988); We thank Peter Arnold for an illuminating discussion of this example.

7. J. Kogut and L. Susskind, Phys. Rev. D11, 1199 (1975).

8. T. Brelidze and H. Thacker (in preparation).

9. W. Bardeen (private communication). 